\title{
MedienPädagogik
}

Zeitschrift für Theorie und Praxis der Medienbildung www.medienpaed.com

Themenheft Nr. 28: Tagungsband: Bildung gemeinsam verändern: Diskussionsbeiträge und Impulse aus Forschung und Praxis. Herausgegeben von David Meinhard, Valentin Dander, Andrea Gumpert, Christoph Rensing, Klaus Rummler und Timo van Treeck.

\section{Normative Professionalisierung freier Journalisten Eine berufspädagogische Perspektive}

Armin Himmelrath

\begin{abstract}
Zusammenfassung
In Zeiten von Fake-News, «Lügenpresse»-Vorwürfen und Diskussionen darüber, wie qualitativ gute Medienarbeit aussehen kann und soll, steigt der Druck auf journalistische Akteure, sich mit berufsethischen Fragen zu befassen. Wer eine entsprechende berufsfachliche Ausbildung durchlaufen hat, ist mit solchen Themen zwangsläufig in Berührung gekommen. Doch die zunehmende Zahl von Freiberuflern ohne entsprechende Fachausbildung, verbunden mit dem immer leichteren Zugang zu digitalen journalistischen Produktionsmitteln und Veröffentlichungswegen, sorgt dafür, dass viele Journalisten über herkömmliche berufsethische Qualifikationsmassnahmen wie z. B. Kurse im Rahmen eines Volontariats oder Fachstudiums gar nicht mehr erreicht wurden bzw. werden. Es stellt sich daher die Frage, wie innerhalb dieser Gruppe von Medienakteuren in Zukunft berufsethische Werte vermittelt werden können und welche Bedingungen einen Prozess ethischer Reflexion unter freien Journalisten/-innen befördern.

Erste Umfrageergebnisse innerhalb der Berufsgruppe legen nahe, dass vor allem Aktivitäten von Redaktionen und Verlagen im Sinne einer schärferen Normierung als adäquate Reaktion auf bestehende berufsethische Defizite gesehen werden. Nachhaltig Erfolg versprechender könnten jedoch Massnahmen sein, die auf die Eigenaktivität und intrinsische Motivation freier Journalisten/-innen setzen. Hier stellt sich die Frage der effektiven Lernund Vermittlungswege. Sie sollten, dem digital geprägten Arbeitsumfeld der Zielgruppe angemessen, auf digitalen Lehr- und E-Learning-Konzepten beruhen.
\end{abstract}

Normative professionalisation of freelance journalist. A perspective of professional education and training

\begin{abstract}
In times of fake news, "Lügenpresse»-presumptions and discussions about how good media work should look like, the pressure on journalistic actors to deal with professional ethics is increasing. Those who have undergone appropriate professional training during their qualification have come into contact with such ethical topics. However, the increasing number of freelancers without corresponding professional training, combined with the easier access to digital journalistic production and dissemination resources, leads to a
\end{abstract}

Himmelrath, Armin. 2017. «Normative Professionalisierung freier Journalisten. Eine berufspädagogische Perspektive». MedienPädagogik 28, (24. Februar), 36-44. https://doi.org/10.21240/mpaed/28/2017.02.24.X. 
situation that many journalists are not able to make use of traditional professional ethical qualification measures, e. g. courses in the context of a trainee program or during their university studies. Therefore, it is necessary to think about new ways of teaching these media professionals about professional values in the future and about the conditions that are supportive for processes of ethical reflection among freelance journalists.

First results suggest that freelancers see foremost activities of editors and publishers in terms of a more restrictive professional normalization to be an adequate answer to their lack of professional ethical reflection. But compared to that, measures that rely on the intrinsic activity and motivation of freelance journalists seem to be more sustainable and promising for this process. This raises the issue of effective ways of learning and teaching. The measures should be based on digital teaching and e-learning concepts, depending on the digitally defined work environment of the target group.

\section{Einleitung}

Die Arbeitswelt in der postmodernen Gesellschaft ist starken Veränderungen unterworfen, unternehmerische Risiken werden zunehmend auf den Einzelnen übertragen (vgl. Kaiser et al. 2012). Im Journalismus sind durch diese Entwicklung deutliche Tendenzen einer Deprofessionalisierung zu beobachten (vgl. Gertler 2013, 5), da zwei Trends zusammenfallen: das zunehmende Outsourcing journalistischer Dienstleistungen aus den Redaktionen heraus einerseits und der - bedingt durch die Digitalisierung - zunehmend leichtere Zugang zu Arbeitsmitteln und Veröffentlichungen für Autoren/-innen andererseits.

Normative Professionalisierung meint die Definition und die Vermittlung berufsethischer Werte und Kenntnisse im Hinblick auf spezifische Berufsfelder. Für den Journalismus fand die systematische Vermittlung dieser Werte bisher im Rahmen der formalisierten Ausbildungswege (redaktionelles Volontariat, journalistisches Fachstudium oder Journalistenschule) statt (vgl. hierzu: Deutscher Presserat 2015). Mit der abnehmenden Bedeutung formal definierter berufsqualifizierender Zugangswege stellt sich jedoch die Frage, inwiefern andere Vermittlungswege genutzt werden können und müssen, um (freien) Journalisten/-innen ein als sinnvoll erachtetes berufsethisches Wertegerüst an die Hand zu geben.

Meyers et al. (2012) konstatieren vor diesem Hintergrund einen grundsätzlichen Perspektivwechsel auf die Definition journalistischer Arbeit: «We (...) shift the focus from whether the person is journalist to whether the work satisfies the conditions that characterize legitimate journalism» (Meyers et al. 2012, 189). Diese Verschiebung birgt aus ethischer Sicht die Gefahr einer Qualitätsreduktion, bietet aber auch die Chance einer neuen Verankerung eines umfassend verstandenen Qualitätsbegriffs im Sinne «guter Arbeit» (Kunneman 2012, 4): Weil die berufspädagogischen StandardAusbildungswege also an Bedeutung verlieren, ethische Standards aber weiterhin 
eingefordert werden, müssen solche Qualifizierungsmassnahmen zur Vermittlung normativer Professionalisierungsinhalte im Sinne einer Berufsethik für den Journalismus über eine neu zu konzipierende, direkte Ansprache der Zielgruppe initiiert werden. Zu den eher unrealistisch erscheinenden Optionen gehören hier eine (an bisherigen Ausbildungskonzepten orientierte) Nachqualifizierung bei Präsenzseminaren oder ein verpflichtender Berufsethik-Nachweis durch die Auftragnehmer gegenüber den Redaktionen. Wie also kann ein Erfolg versprechendes Qualifikationsbzw. Schulungskonzept aussehen? Wer sollte es mit welchen Inhalten und für welche Bedürfnisse anbieten? Und mit welchen Medien lassen sich die gewünschten Inhalte in die Zielgruppe hinein vermitteln? Da Journalismus und Medien längst ein durchdigitalisiertes Arbeitsfeld sind, gehe ich von der Hypothese aus, dass E-LearningAngebote in unterschiedlicher Form oder zumindest stark digital geprägte BlendedLearning-Konzepte die grössten Erfolgsaussichten haben. Dies zu überprüfen und die aufgeworfenen Fragen zu beantworten, ist das Ziel meiner Dissertation an der Humanistischen Universität Utrecht (UvH). Die Forschungsarbeit ist im UvH-Forschungscluster «Normative Professionalisierung» angesiedelt.

\section{Projektbeschreibung und erste Ergebnisse}

Meine Forschungsarbeit geht der Frage nach, welche Bedingungen einen kontinuierlichen Prozess ethischer Reflexion unter freien Journalisten/-innen unter Berücksichtigung der Rahmenbedingungen ihrer freiberuflichen Arbeit fördern können und wie das Bewusstsein bzw. die Motivation für ethische Fragen bei diesen Medienakteuren/innen geschärft werden kann. Dazu gehört zwingend auch die Frage nach der Form der Vermittlung: Durch welche Art von Qualifizierungsangeboten lassen sich freie Journalisten/-innen ansprechen und motivieren, sich mit berufsethischen Fragen zu befassen? Wie müssen diese Angebote medial aufbereitet sein - konkreter: Welche Rolle kann E-Learning in diesem Zusammenhang spielen?

Im Rahmen der Mixed-Methods-Studie werden freie Journalisten/-innen sowie journalistische Auftraggeber/innen in Redaktionen in einem ersten Schritt per Fragebogen danach befragt, inwieweit sie einen Mangel im Bereich der berufsethischen Kompetenz/Ausbildung der Freelancer wahrnehmen und welche Gegenmassnahmen sie ggf. vorschlagen. Die Ergebnisse werden in qualitativen Experten/-innen-Interviews (offene Leitfragen-Interviews) und in Gruppendiskussionen mit Stakeholdern debattiert und weiter entwickelt. Die dabei entstehenden Ergebnisse sollen dann zu einem zweiten Fragebogen verdichtet werden, um die gefundenen Resultate noch einmal rückzukoppeln und zu überprüfen. 
In einer ersten Erhebung ${ }^{1}$ wurde zunächst die Relevanz des Themas bei freien Journalisten/-innen auf qualitativer Ebene überprüft und bestätigt: $84,89 \%$ der TN stimmten der Aussage zu, dass ethische Überlegungen in ihrer täglichen journalistischen Arbeit «eine wichtige Rolle» spielen. 60,47\% allerdings widersprachen der Aussage, die Bedeutung ethischer Qualifikation im Journalismus sei «in den vergangenen Jahren deutlich wichtiger geworden». Und 26,75\% stimmten der Aussage eher oder ganz zu, dass journalistisch-ethische Fragen im persönlichen Arbeitsalltag «kaum berücksichtigt» werden.

Die Auswertung der im Fragebogen erhobenen offenen Antworten zu Frage 8 («Wie könnte Ihrer Meinung nach die [berufs-]ethische Reflexion freier Journalistinnen und Journalisten am besten gefördert werden?») erfolgte nach Mayring $(2000,2007)$, Mayring und Gläser-Zikuda (2007) qualitativ inhaltsanalytisch. Die Kategorienentwicklung wurde dabei anhand des Materials induktiv vorgenommen. Leitkategorie im ersten Auswertungsschritt war dabei die Frage, welche Akteure aus Sicht der befragten freien Journalisten/-innen primär durch ihre Aktivitäten zu einer besseren berufsethischen Reflexion beitragen sollten. In einer zweiten Auswertungsrunde wurden dann Kategorien zur Art der gewünschten/geforderten Aktivitäten gebildet. Ziel war es dabei, inhaltliche Kriterien und Aspekte zu entwickeln, die für die folgenden qualitativen Experteninterviews und Gruppengespräche als Diskussionsanlässe genutzt werden können. Die gegebenen freien Antworten zu dieser Frage ( $n=61$; alle von freien Journalisten/-innen) wurden dementsprechend zunächst daraufhin ausgewertet, wer von den Teilnehmern/-innen als verantwortlicher Akteur möglicher reflexionsverbessernder Massnahmen angesprochen wurde (vgl. Tab. 1).

\begin{tabular}{|l|l|l|l|}
\hline & Eigenaktivität & $\begin{array}{l}\text { Fremdaktivität durch } \\
\text { Auftraggeber }\end{array}$ & $\begin{array}{l}\text { Fremdaktivität durch } \\
\text { externe Dritte }\end{array}$ \\
\hline Akteur & freie Journalisten & $\begin{array}{l}\text { Redaktionen, Verlage, } \\
\text { Sender, Auftraggeber }\end{array}$ & $\begin{array}{l}\text { z. B. Presserat, Fachzeit- } \\
\text { schriften, Berufsverbän- } \\
\text { de, «Öffentlichkeit» }\end{array}$ \\
\hline Beispiele & $\begin{array}{l}\text { Weiterbildung, Selbstver- } \\
\text { pflichtung, Netzwerkbil- } \\
\text { dung etc. }\end{array}$ & $\begin{array}{l}\text { finanzielle Kapazitäten, } \\
\text { Standards, Aus- und Wei- } \\
\text { terbildung, Sanktionen } \\
\text { etc. }\end{array}$ & $\begin{array}{l}\text { Preise für best practice, } \\
\text { Feedback durch Öffent- } \\
\text { lichkeit }\end{array}$ \\
\hline Code & E & FA & FE \\
\hline
\end{tabular}

Tab. 1.: Antwortkategorien der Akteure zu Frage 8.

1 netzbasierter Fragebogen, Mai-August 2015, n=81. 
Die gewählte Kategorisierung erwies sich als konsistent. Im Ergebnis der ersten Auswertung (Tab. 2) zeigt sich damit eine starke Betonung der Forderung, dass sich die Auftraggeber (Redaktionen, Sender, Verlage etc.) als verantwortliche Akteure bei der Förderung der berufsethischen Reflexion freier Journalisten/ -innen engagieren sollten.

Dies gilt umso mehr, wenn man zu den 36 Nennungen, die alleine auf die Auftraggeber abzielen, noch die 16 Nennungen hinzunimmt, in denen die Auftraggeber zusammen mit weiteren potenziellen Akteuren benannt werden.

Die zweithäufigste Nennung betrifft die Eigenaktivität der freien Journalisten/-innen, die fünf Mal alleine sowie $13 \mathrm{Mal}$ in Zusammenhang mit anderen Akteuren genannt werden.

\begin{tabular}{|l|l|l|}
\hline $\begin{array}{l}\text { Kategorie } \\
\text { Akteur }\end{array}$ & Anzahl & Antworten Nr. \\
\hline FA & 36 & $\begin{array}{l}6,7,9,13,15,18,19,21,22,23,24,25,26,27,29,30,31,32,33,34,37, \\
40,41,42,43,44,45,47,48,49,54,55,57,59,60,61\end{array}$ \\
\hline FA+ E & 12 & $1,2,3,12,16,17,35,39,46,52,53,56$ \\
\hline E & 5 & $5,8,10,14,36$ \\
\hline FE & 3 & $28,38,50$ \\
\hline FA + FE & 3 & $11,20,58$ \\
\hline FA + FE + E & 1 & 4 \\
\hline sonstige & 1 & 51 \\
\hline gesamt & 61 & \\
\hline
\end{tabular}

Tab. 2.: Ergebnis der Kategorisierung der Antworten auf Frage 8 nach Akteuren ( $E$ = Eigenaktivität, FA = Fremdaktivität Auftraggeber, FE = Fremdaktivität externer Akteur).

Der starke Fokus auf mögliche Aktivitäten der Auftraggeber lässt sich hier möglicherweise mit der Stichprobe erklären: Es handelt sich bei allen Teilnehmern/-innen um freie Journalisten/-innen. Zu überprüfen wäre, ob eine Stichprobe mit ausschliesslich angestellten Redakteuren/-innen zu einem gespiegelten Ergebnis führen würde, ob also angestellte Journalisten/-innen ihrerseits die Handlungsoptionen für Aktivitäten zur berufsethischen Reflexion vor allem bei den Freien sehen.

Dennoch lässt sich aus dieser ersten Kategorisierung der inhaltliche Diskussionsimpuls ableiten, dass zumindest freie Journalisten/-innen offenbar die grösseren Handlungspotenziale im Hinblick auf bessere Rahmenbedingungen für eine ethische Reflexion der Freien auf Seiten der Auftraggeber und in den Redaktionen sehen. $\mathrm{Zu}$ fragen wäre demnach, ob die interviewten Experten/-innen diese Einschätzung teilen oder ihr widersprechen und mit welchen Argumenten sie dies tun. 
In der genaueren Durchsicht des Materials zeigte sich jedoch schnell, dass die Kategorisierung nach adressierten Akteuren für reflexionsverbessernde Massnahmen zu grob ist bzw. dass die Antworten der Teilnehmer/innen noch weitere inhaltliche Anregungen enthalten. In einem zweiten Analysedurchgang wurden die vorliegenden Texte deshalb Kategorien inhaltlicher Handlungsoptionen zugeordnet. Während in der ersten Analyse die potenziellen Akteure im Mittelpunkt standen (Wer sollte etwas tun?), waren es in der zweiten Runde die Handlungsoptionen (Was kann getan werden?). Bei diesen Kategorien handelt es sich um:

\begin{tabular}{|l|l|l|}
\hline Kategorie & Beispiele & Code \\
\hline $\begin{array}{l}\text { Eigeninitiative freier } \\
\text { Journalisten }\end{array}$ & $\begin{array}{l}\text { Selbstverpflichtung, Code of Conduct, Netzwerkbildung } \\
\text { etc. }\end{array}$ & EI \\
\hline $\begin{array}{l}\text { Zusammenarbeit Freie } \\
\text { - Auftraggeber }\end{array}$ & Treffen, Feedbackkultur, Absprachen, Briefings etc. & ZU \\
\hline $\begin{array}{l}\text { (mehr) Geld und Zeit } \\
\text { als Ressourcen }\end{array}$ & mehr Honorar, weniger Zeitdruck etc. & GE \\
\hline $\begin{array}{l}\text { Sanktionen durch Auf- } \\
\text { traggeber }\end{array}$ & Nicht-Abdruck, Strafen etc. & SA \\
\hline $\begin{array}{l}\text { Redaktionelle Stan- } \\
\text { dards }\end{array}$ & Ethik-Regelwerk einer Redaktion etc. & RS \\
\hline Best Practice & Preise/ Auszeichnungen für gute Arbeiten etc. & BP \\
\hline $\begin{array}{l}\text { Öffentliche Kontrolle/ } \\
\text { Debatte }\end{array}$ & $\begin{array}{l}\text { öffentliche Diskussion, Publikationen über journalistische } \\
\text { Ethik etc. }\end{array}$ & ÖK \\
\hline Ausbildung & Volontariat, Studium & AB \\
\hline Weiterbildung & Seminare, Vorträge etc. & WB \\
\hline
\end{tabular}

Tab. 3.: Antwortkategorien Handlungsoptionen zu Frage 8 und Codierung.

Die gewählte Kategorisierung erwies sich als praktikabel. Die Ausdifferenzierung insbesondere der Handlungsoptionen von Auftraggebern ist den Ergebnissen der ersten Analyse geschuldet, in der ja gerade die Auftraggeber als potenzielle Akteure aus Sicht der Teilnehmer/-innen herausgearbeitet wurden. Da zahlreiche Antworten der Teilnehmer/innen mehrere Handlungsoptionen benannten, wurden entsprechende Stichworte und Schlagwörter jeweils einer der oben genannten Kategorien zugeordnet. Einzelne Antworten kamen damit auf bis zu sieben Kategoriezuordnungen (Tab. 4). 


\begin{tabular}{|l|l|l|}
\hline $\begin{array}{l}\text { Kategorie } \\
\text { Handlungs- } \\
\text { option }\end{array}$ & Anzahl & Antworten Nr. \\
\hline RS & 20 & $2,3,6,12,15,19,21,23,25,29,31,34,39,43,44,45,46,49,52,61$ \\
\hline ZU & 19 & $3,4,7,10,11,12,16,17,18,23,24,35,40,41,45,48,52,53,55$ \\
\hline WB & 18 & $1,2,3,4,7,9,11,13,16,24,25,26,27,30,32,37,42,56$ \\
\hline GE & 13 & $1,3,4,15,33,34,39,41,46,47,54,57,59$ \\
\hline EI & 12 & $2,3,4,5,10,14,16,36,39,46,53,56$ \\
\hline ÖK & 9 & $8,12,14,20,28,47,49,50,58$ \\
\hline AB & 7 & $4,7,22,23,37,58,59$ \\
\hline SA & 6 & $4,19,29,31,56,60$ \\
\hline BP & 2 & 4,53 \\
\hline sonstige & 2 & 38,51 \\
\hline
\end{tabular}

Tab. 4.: $\quad$ Ergebnis der Kategorisierung der Antworten auf Frage 8 nach Handlungsoptionen (RS = Redaktionelle Standards, ZU = Zusammenarbeit, WB = Weiterbildung, GE = Geld $/$ Ressourcen, $\mathrm{EI}=$ Eigeninitiative, $\mathrm{ÖK}=$ Öffentliche Kontrolle, $\mathrm{AB}=$ Ausbildung, $\mathrm{SA}=$ Sanktionen, $\mathrm{BP}=$ Best Practice).

Folgt man den Überlegungen von Meyers et al. (2012), dann werden in Zukunft vor allem die Berufsverbände für "professional journalism» (Meyers et al. 2012, 193) eine zunehmend wichtige Rolle spielen, die die Medienakteure dabei unterstützen, eine selbstorganisierte, freiwillige Form von Professionalisierung voranzutreiben.

Journalism can adopt a voluntary designation, either granted by an agency or self-declared, that identifies the individual as properly trained and committed [...] Journalists need to find ways to guarantee high standards that are not controlled by their employing organizations. We suggest some collective strategies for acquiring and asserting more power to exercise control over good work modeled on the labor movement. (Meyers et al., 193)

Die Argumentation folgt hier eng Sennetts Begriff von «Handwerk» (vgl. Sennett 2008, 31ff.) und qualitätsorientierter Arbeit (Sennett 2008, 321ff.) als dem Impuls, eine gewählte Profession um ihrer selbst willen gut auszuführen. Gleichzeitig bringen Meyers et al. mit den journalistischen Gewerkschaften Akteure ins Spiel, die als Träger bzw. Anbieter von ethischen Qualifikationsmassnahmen von beiden Seiten Redakteuren/-innen einerseits und freien Journalisten/-innen andererseits - als extern Handelnde betrachtet werden können und deshalb möglicherweise Lehr- und Lernprozesse leichter initiieren können als die beteiligten Stakeholder selbst. 


\section{Reflexion und Ausblick}

Im Ergebnis zeigte sich, dass zwar redaktionelle Standards und damit eine Handlungsoption alleine auf Seiten der Auftraggeber am häufigsten benannt wurden, dass aber Handlungsmöglichkeiten unter Einbeziehung der freien Journalisten/-innen ebenfalls häufige Nennungen zu verzeichnen hatten.

Die dabei erwähnten Aspekte der besseren Zusammenarbeit mit Auftraggebern, insbesondere aber auch der kontinuierlichen eigenen Weiterbildung deuten nicht nur auf eine ausgeprägte Bereitschaft zur Beschäftigung mit berufsethischen Fragen hin, sondern werfen - zumal vor dem Hintergrund der Nennung von Geld, Zeit, Ressourcen als wichtigen journalistischen Alltagsdeterminanten - die Frage auf, inwieweit digital zur Verfügung gestellte Aus- und Weiterbildungsangebote eine zielführende Massnahme sein könnten, um einen kontinuierlichen Prozess ethischer Reflexion unter freien Journalisten/-innen zu fördern. Die bisher erhobenen Daten erlauben dazu keine Aussage; die Frage wird daher in den kommenden Experteninterviews eine Rolle spielen.

Wie für das Berufsfeld Journalismus dabei die als notwendig erachteten ethischen Prinzipien vermittelt werden können und welche Rolle dabei Aspekte etwa der digital gestützten Beschäftigung mit bestimmten Fragestellungen und Themenkomplexen spielen könnten, soll daher im weiteren Fortgang des Forschungsprojekts untersucht werden.

\section{Literatur}

Deutscher Presserat. 2015. Publizistische Grundsätze (Pressekodex). Richtlinien für die publizistische Arbeit nach den Empfehlungen des Deutschen Presserats. http://www.presserat.de/ fileadmin/user_upload/Downloads_Dateien/Pressekodex_bo_web_2015.pdf.

Friedrichsen, Mike, und Martin Gertler. 2011. Medien zwischen Ökonomie und Qualität: Medienethik als Instrument der Medienwirtschaft. (Schriften zur Medienwirtschaft und zum Medienmanagement. Band 24). Baden-Baden: Nomos Verlagsgesellschaft.

Gertler, Martin. 2013. «Meaning-generating propositions of reality by media. Quality attributes and functions of journalism.» Journal of Information, Communication and Ethics in Society, $11(1): 4-18$.

Kaiser, Stephan, Stefan Süß, und Ingrid Josephs. 2012. Freelancer als Forschungsgegenstand und Praxisphänomen. Betriebswirtschaftliche und psychologische Perspektiven. Frankfurt am Main: Peter Lang.

Krippendorff, Klaus. 2004. Content Analysis: An Introduction to Its Methodology (2. Auflage). Thousand Oaks: Sage.

Kunneman, Harry, Hrsg. 2012. Good Work. The Ethics of Craftmanship. Amsterdam: SWP Publishers. 
Mayring, Philipp. 2000. "Qualitative Inhaltsanalyse». Forum Qualitative Sozialforschung / Forum: Qualitative Social Research, 1(2). http://www.qualitative-research.net/index.php/fqs/ article/download/1089/2384.

Mayring, Philipp, und Michaela Gläser-Zikuda, Hrsg. 2005. Die Praxis der qualitativen Inhaltsanalyse. Weinheim und Basel: Beltz Verlag.

Mayring, Philipp. 2007. Qualitative Inhaltsanalyse. Grundlagen und Techniken (9. Auflage). Weinheim: Deutscher Studien Verlag.

Meyers, Christopher, Wendy N. Wyatt, Sandra L. Borden, und Edward Wasserman. 2012. «Professionalism, not professionals.» Journal of Mass Media Ethics, 27 (3):189-205. doi:10.1080 /08900523.2012.700212.

Schreier, Margrit. 2012. Qualitative Content Analysis in Practice. London: Sage Publications.

Sennett, Richard. 2008. Handwerk. Berlin: Berlin Verlag.

\section{Tabellen}

Tab. 1.: Antwortkategorien der Akteure zu Frage 8.

Tab. 2.: Ergebnis der Kategorisierung der Antworten auf Frage 8 nach Akteuren ( $E=$ Eigenaktivität, $\mathrm{FA}=$ Fremdaktivität Auftraggeber, $\mathrm{FE}=$ Fremdaktivität externer Akteur).

Tab. 3.: Antwortkategorien Handlungsoptionen zu Frage 8 und Codierung.

Tab. 4.: Ergebnis der Kategorisierung der Antworten auf Frage 8 nach Handlungsoptionen (RS = Redaktionelle Standards, ZU = Zusammenarbeit, WB = Weiterbildung, GE = Geld $/$ Ressourcen, $\mathrm{EI}=$ Eigeninitiative, $\mathrm{ÖK}=$ Öffentliche Kontrolle, $\mathrm{AB}=$ Ausbildung, $\mathrm{SA}=$ Sanktionen, $\mathrm{BP}=$ Best Practice). 